\title{
Featureless quantum paramagnet with frustrated criticality and competing spiral magnetism on spin-1 honeycomb lattice magnet
}

\author{
Jian Qiao Liu, ${ }^{1,2}$ Fei-Ye Li, ${ }^{2,3}$ Gang Chen $\odot,{ }^{2,3,4, *}$ and Ziqiang Wang ${ }^{5}$ \\ ${ }^{1}$ International Center for Quantum Materials, School of Physics, Peking University, Beijing 100871, China \\ ${ }^{2}$ Department of Physics and HKU-UCAS Joint Institute for Theoretical and Computational Physics at Hong Kong, \\ The University of Hong Kong, Hong Kong, China \\ ${ }^{3}$ State Key Laboratory of Surface Physics and Department of Physics, Institute of Nanoelectronics and Quantum Computing, \\ Fudan University, Shanghai 200433, China \\ ${ }^{4}$ Collaborative Innovation Center of Advanced Microstructures, Nanjing University, Nanjing 210093, China \\ ${ }^{5}$ Department of Physics, Boston College, Chestnut Hill, Massachusetts 02467, USA
}

(Received 1 May 2020; accepted 29 July 2020; published 18 August 2020)

\begin{abstract}
We study the spin-1 honeycomb lattice magnets with frustrated exchange interactions. The proposed microscopic spin model contains first- and second-neighbor Heisenberg interactions as well as the single-ion anisotropy. We establish a rich phase diagram that includes a featureless quantum paramagnet and various spin spiral states induced by the mechanism of order by quantum disorder. Although the quantum paramagnet is dubbed featureless, it is shown that the magnetic excitations develop a contour degeneracy in the reciprocal space at the band minima. These contour degenerate excitations are responsible for the frustrated criticality from the quantum paramagnet to the ordered phases. This work illustrates the effects of magnetic frustration on both magnetic orderings and the magnetic excitations. We discuss the experimental relevance to various Ni-based honeycomb lattice magnets.
\end{abstract}

DOI: 10.1103/PhysRevResearch.2.033260

\section{INTRODUCTION}

Frustrated magnetism is a large topic in modern strong correlation physics [1-3]. Frustration usually refers to competing interactions that cannot be optimized simultaneously. For magnetic systems, these interactions are the exchange interactions between the local magnetic moments. The Ising interaction on the triangular lattice is often used as an example to illustrate the concept of frustration [1]. Due to the strong magnetic frustration, conventional magnetic orders are often expected to be suppressed. Instead, unconventional quantum phases such as quantum spin liquids [4-6], skyrmion lattices and spin nematics [7-9], and exotic excitations such as spinons, topological magnons [10-13] and magnetic monopoles [14-16], may emerge. Despite being an important notion in modern condensed matter physics, magnetic frustration does not seem to have a strong phenomenological correspondence or a mathematical characterization. This is quite different from other contemporary notions such as emergent symmetry [17], topology [18-20], and entanglements [21]. By comparison, magnetic frustration is more like the physical origin or the driving force for unconventional

\footnotetext{
*gangchen.physics@gmail.com

Published by the American Physical Society under the terms of the Creative Commons Attribution 4.0 International license. Further distribution of this work must maintain attribution to the author(s) and the published article's title, journal citation, and DOI.
}

magnetic properties, rather than a description of the internal structures and the physical consequences. Thus we are more interested in the understanding of various physical consequences that result from the magnetic frustration in magnetic systems.

Empirically, magnetic frustration could lead to a large number of degenerate or nearly degenerate low-energy states such that the system has a difficulty to develop a conventional magnetic order and the ordering temperature is often suppressed. An empirical parameter, dubbed "frustration parameter" [3], is thus used to characterize the level of frustration

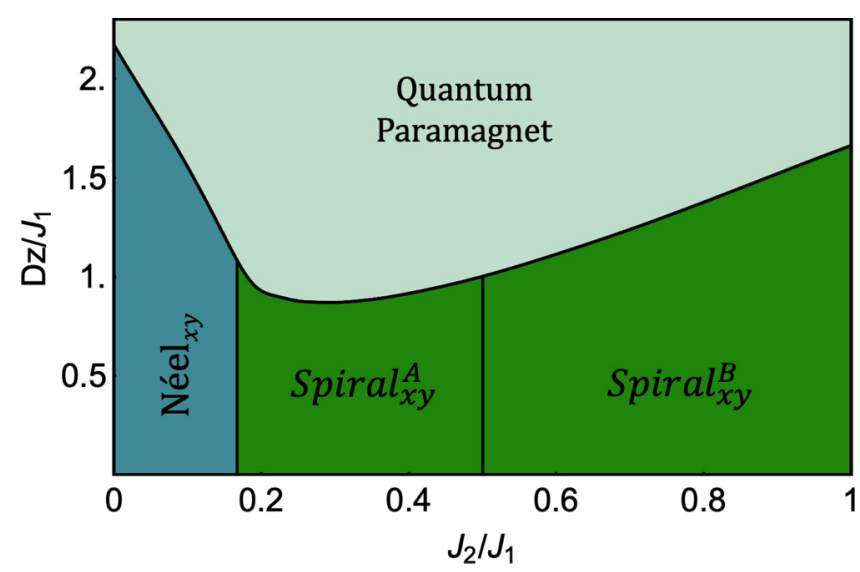

FIG. 1. Ground-state phase diagram of the $J_{1}-J_{2}-D_{z}$ model. The details of each phase are explained in the main text. 
of the system. The "frustration parameter" is defined as the ratio between the Curie-Weiss temperature and the ordering temperature. The larger the "frustration parameter" is, the more frustrated the system is. This simple empirical parameter, however, does not actually provide much information or understanding about the low-energy physical properties of the system. For the low-energy physical properties, one can then focus on the ground-state phases and the corresponding emergent physics associated with the quantum phases. These emergent physics are usually controlled by the quantum phases rather than being connected to the magnetic frustration in a direct fashion. Therefore it seems that the magnetic frustration merely works as an empirical route to look for unconventional quantum phases with spin degrees of freedom. In this paper, we work on the specific spin-1 honeycomb magnet and show that the emergent low-energy magnetic properties are directly tied to the magnetic frustration in this system.

Spin-1/2 honeycomb lattice magnets, especially the honeycomb Kitaev materials [22-24], have attracted a lot of attention in the field. The spin-orbital entanglement of the local moments brings rather anistropic interactions between neighboring spins and creates a strong frustration and a disordered Kitaev spin liquid ground state even for a geometrically unfrustrated honeycomb lattice [25]. Recent efforts try to extend spin-1/2 Kitaev materials to high-spin magnets, mainly spin-1 magnets, where the heavy ligand atoms may bring some anisotropic spin interactions through the exchange path [26]. Although exact solutions are not available for high spin Kitaev materials, it is hoped that exotic quantum state may still persist to high spin systems, especially since quantum effect in spin-1 magnets is still strong and magnetic frustration could further enhance it. Moreover, it is well-known that, with larger physical Hilbert space, the spin-1 magnet could bring more distinct physics from the spin-1/2 magnet. This has been well illustrated in the strong (topological) distinction between the spin-1/2 Heisenberg chain and spin-1 Haldane chain [27]. Compared to the tremendous efforts in various spin-1/2 magnets, the attention in spin-1 magnets is rather limited. Thus it is particularly timely to explore the potentially rich physics of frustrated spin-1 magnets [28-36].

On the experimental side, several new materials have been proposed as spin-1 honeycomb magnets and show distinct magnetic properties. All of them are Ni-based and have either a honeycomb layer structure or a buckled honeycomb structure with an A-B stacked triangular bilayer. Inspired by the growing interest in the spin-1 magnets and the existing experiments on spin-1 honeycomb magnets, we propose and study a minimal model for spin-1 local moments on a honeycomb lattice with the Hamiltonian

$$
H=J_{1} \sum_{\langle i j\rangle} S_{i} \cdot S_{j}+J_{2} \sum_{\langle\langle i j\rangle\rangle} S_{i} \cdot S_{j}+D_{z} \sum_{i}\left(S_{i}^{z}\right)^{2},
$$

where $S_{i}, S_{j}$ are spin-1 local moments on the honeycomb lattice, $\langle i j\rangle$ denotes exchange interactions of the first-neighbor spin pairs, $\langle\langle i j\rangle\rangle$ denotes exchange interactions of the second neighbor pairs. We work on the regime with $J_{1}>0$ and $J_{2}>0$, and a ferromagnetic $J_{1}$ is obtained by applying time reversal transformation on one sublattice. Here we are mostly interested in the Heisenberg spin degrees of freedom. We introduce a single-ion spin anisotropy that is generically allowed by the planar lattice geometry and the large spin moment. This single-ion spin anisotropy is necessary for the materials that show a clear in-plane and out-plane spin anisotropy. We are interested in the easy-plane regime with $D_{z}>0$ for the model. It is readily seen that the easy-axis regime is connected to the Ising limit where the quantum effect can be suppressed. In our analysis, we start from the well-defined limit with a strong easy-plane anisotropy. In this limit, the ground state is clearly known as a trivial and featureless quantum paramagnet with $\left|S_{i}^{z}=0\right\rangle$ on each site. As we show in this paper, although this quantum paramagnet is dubbed "trivial and featureless," the magnetic frustration brings extra features on top of this featureless ground state. The coherent magnetic excitations develop a contour degenerate band minima when the frustration of the exchange interaction becomes large. We identify this property as one direct consequence of the magnetic frustration. As the single-ion anisotropy becomes weaker, the gap of the magnetic excitation is reduced and eventually becomes zero, and the system develops magnetism. It is the degenerate low-enegy magnetic excitations that are responsible for the critical fluctuations and the development of the magnetism. We further show the unusual critical properties due to these critical modes in the vincinity of the phase transition between the featureless quantum paramagnet and the ordered phases. This is again linked to the magnetic frustration. On the ordered side, the ordering structure of the system is directly related to the degenerate low-energy band minima in the quantum paramagnet and the critical modes. We show, quantum fluctuations are needed to break the degeneracy among the candidate ordering wave vectors on the degenerate contours in the momentum space. The consequence of this order by quantum disorder is explained. The full phase diagram of the model is summarized in Fig. 1.

The remaining part of the paper is organized as follows. In Sec. II, we study the featureless quantum paramagnet with a large single-ion anisotropy and point out the nontrivial features in the magnetic excitations. In Sec. III, we consider the instabilities of the featureless quantum paramagnet and study the critical properties of the frustrated quantum criticality. In Sec. IV, we focus on the ordered regime and explain the magnetic orders from the mechanism of order by quantum disorder. Finally in Sec. V, we discuss various experimental relevance to the $\mathrm{Ni}$-based honeycomb lattice magnets.

\section{II. “FEATURELESS” QUANTUM PARAMAGNET}

We start from the strong single-ion limit where there is a well-defined ground state to work with. When $D_{z} \gg J_{1}, J_{2}$, the ground state is a trivial quantum paramagnet and is approximated by

$$
\left|\Psi_{\mathrm{GS}}\right\rangle=\prod_{i}\left|S_{i}^{z} \equiv 0\right\rangle .
$$

It is well-known that this trivial state has no order of any kind and preserves all the symmetries of the original Hamiltonian. Thus it is simply featureless. As we will show below, however, the magnetic excitation of this featureless state develops some extra features when the system becomes frustrated. This property may be interpreted as the physical consequence of the frustration on this featureless state. Since this state does 
not have any conventional magnetic order, the conventional spin wave theory cannot be applied to compute the magnetic excitation with respect to this featureless state. Here, we adopt the flavor wave theory $[30,37,38]$. This theory was originally developed for the interacting spin-orbital local moments with an effective fundamental $\mathrm{SU}(4)$ representation on each site of the triangular lattice. This theory is not only applicable to the featureless ground state for the spin-1 magnets, but also applies to any kind of product states where the ground state can be separated into the direct product of local states for local sites or local cluster units.

The spirit of the flavor wave theory is similar to the Schwinger boson parton representation of the spin variables. Here, one introduces three boson operators for the three states of the spin-1 Hilbert space and condenses one of them to generate the featureless quantum paramagnetic state. Henceforth, the remaining two auxiliary bosons describe the magnetic excitations. The advantage of the flavor wave approach is that the physical nature of the ground state and the corresponding excitations is straight-forward. For this purpose, we define the mapping between the boson operators and the spin states as

$$
\begin{aligned}
a_{i, 1}^{\dagger}|\emptyset\rangle & \equiv\left|S_{i}^{z}=1\right\rangle, \\
a_{i, 0}^{\dagger}|\emptyset\rangle & \equiv\left|S_{i}^{z}=0\right\rangle, \\
a_{i, 1}^{\dagger}|\emptyset\rangle & \equiv\left|S_{i}^{z}=\overline{1}\right\rangle,
\end{aligned}
$$

where the state $|\emptyset\rangle$ is the vacuum state. The physical spin-one operator, $S_{i}^{\alpha}$, can be written as

$$
S_{i}^{\alpha} \equiv \sum_{m, n}\left\langle S_{i}^{z}=m\left|S_{i}^{\alpha}\right| S_{i}^{z}=n\right\rangle a_{i, m}^{\dagger} a_{i, n}
$$

with $\alpha=x, y, z$ and $m, n=1,0, \overline{1}$. Apparently, this mapping enlarges the physical Hilbert space. Thus we need to impose a constraint to get back to the physical Hilbert space with

$$
a_{i, 1}^{\dagger} a_{i, 1}+a_{i, 0}^{\dagger} a_{i, 0}+a_{i, \overline{1}}^{\dagger} a_{i, \overline{1}} \equiv 1 \text {. }
$$

on each lattice site. In terms of these flavor wave bosons, the exchange part of Eq. (1) is converted into four-boson interacting terms while the single-ion anisotropy is quadratic in the boson operators. To obtain the quantum paramagnetic state in Eq. (2), one simply condenses the $a_{0}$ boson by replacing

$$
\left\langle a_{i, 0}^{\dagger}\right\rangle \approx\left\langle a_{i, 0}\right\rangle \rightarrow\left(1-a_{i, 1}^{\dagger} a_{i, 1}-a_{i, \overline{1}}^{\dagger} a_{i, \overline{1}}\right)^{\frac{1}{2}}
$$

in the Hamiltonian. At the level of linear flavor wave treatment, one keeps the quadratic part of the bosonic operators in the Hamiltonian. This linear flavor wave Hamiltonian is given as

$$
H_{\mathrm{fw}}=\sum_{k \in \mathrm{BZ}}\left(\psi_{k, \mathrm{~A}}^{\dagger} \quad \psi_{k, \mathrm{~B}}^{\dagger}\right) \mathcal{M}(k)\left(\begin{array}{c}
\psi_{k, \mathrm{~A}} \\
\psi_{k, \mathrm{~B}}
\end{array}\right),
$$

where $\psi_{k, \mu} \equiv\left(a_{k, 1, \mu}, a_{k, \overline{1}, \mu}, a_{\bar{k}, 1, \mu}^{\dagger}, a_{\bar{k}, \overline{1}, \mu}^{\dagger}\right)^{\mathrm{T}}$ and $\mu=A, B$ labels the two sublattices of the honeycomb lattice. The Hamiltonian matrix $\mathcal{M}(k)$ is given as

$$
\mathcal{M}(k)=\left(\begin{array}{ll}
\mathcal{M}_{1} & \mathcal{M}_{2} \\
\mathcal{M}_{2}^{*} & \mathcal{M}_{1}
\end{array}\right),
$$

where

$$
\begin{gathered}
\mathcal{M}_{1}=\left(\begin{array}{cccc}
m_{2} & 0 & 0 & m_{2} \\
0 & m_{2} & m_{2} & 0 \\
0 & m_{2} & m_{2} & 0 \\
m_{2} & 0 & 0 & m_{2}
\end{array}\right)+D_{z} \mathcal{I}_{4 \times 4}, \\
\mathcal{M}_{2}=\left(\begin{array}{cccc}
m_{1} & 0 & 0 & m_{1} \\
0 & m_{1} & m_{1} & 0 \\
0 & m_{1} & m_{1} & 0 \\
m_{1} & 0 & 0 & m_{1}
\end{array}\right),
\end{gathered}
$$

and $\mathcal{I}_{4 \times 4}$ is a $4 \times 4$ identity matrix. Here the entries of the matrix are

$$
\begin{aligned}
& m_{1} \equiv J_{1} \sum_{\mu} e^{-i k \cdot b_{\mu}}, \\
& m_{2} \equiv J_{2} \sum_{\mu} e^{-i k \cdot d_{\mu}},
\end{aligned}
$$

where the summations above are over the first-neighbor vectors $\left\{b_{\mu}\right\}$ and the second-neighbor vectors $\left\{d_{\mu}\right\}$ of the honeycomb lattice, respectively.

Using the Bogoliubov transformation, we establish the magnetic excitations from the linear flavor wave Hamiltonian. The dispersions are

$$
\omega_{ \pm}(k)=\sqrt{D_{z}\left(D_{z}+2\left[J_{2}\left(\Lambda^{2}(k)-3\right) \pm J_{1} \Lambda(k)\right]\right)},
$$

where $\Lambda(k) \equiv\left|\sum_{\mu} e^{-i k \cdot b_{\mu}}\right|$, with $\left\{b_{\mu}\right\}$ being three nearest neighbor vectors of the honeycomb lattice. Here both two branches $\omega_{+}(k)$ and $\omega_{-}(k)$ are two-fold degenerate, that is associated to the time reversal symmetry, i.e., the equivalence between the $S_{z}=1$ excitation and the $S_{z}=-1$ excitation. In total, we have $4=2 \times 2$ branches of magnetic excitations, that is consistent with the number of the sublattices and the flavors. This is quite different from the coherent spin wave excitations for a conventional magnetically ordered state where the branch number is equal to the number of the magnetic sublattices.

On the top panel of Fig. 2, we depict the evolution of the band structure for the low-lying mode $\omega_{-}(k)$ in the reciprocal space by varying $J_{2} / J_{1}$. As expected, the flavor wave magnetic excitations are fully gapped. A further examination of the spectrum points to a contour degeneracy at the band minimum. To understand that we realize that the band minimum of $\omega_{-}(k)$ occurs at

$$
\Lambda(k)=\frac{J_{1}}{2 J_{2}},
$$

and the solutions of the above equation determine the positions of the band minima. When $J_{2} / J_{1} \leqslant 1 / 6$, a single band minimum is realized at the $\Gamma$ point. When $J_{2} / J_{1}>1 / 6$, degenerate minima with a contour degeneracy in the reciprocal space are realized. As $J_{2} / J_{1}$ increases from $1 / 6$, the contour first emerges surrounding the $\Gamma$ point, and gradually expands. At $J_{2} / J_{1}=1 / 2$, the contour becomes a perfect hexagon that is formed by connecting the six equivalent $\mathrm{M}$ points at the Brillouin zone boundary. As $J_{2} / J_{1}$ is further increased, the contour surrounds $K$ and $K^{\prime}$ points, and finally the contour shrinks to $K$ and $K^{\prime}$ points when $J_{2} / J_{1} \rightarrow \infty$. The evolution of the degenerate contour is depicted on the lower panel of Fig. 2. The emergence of this degenerate contour arises from 

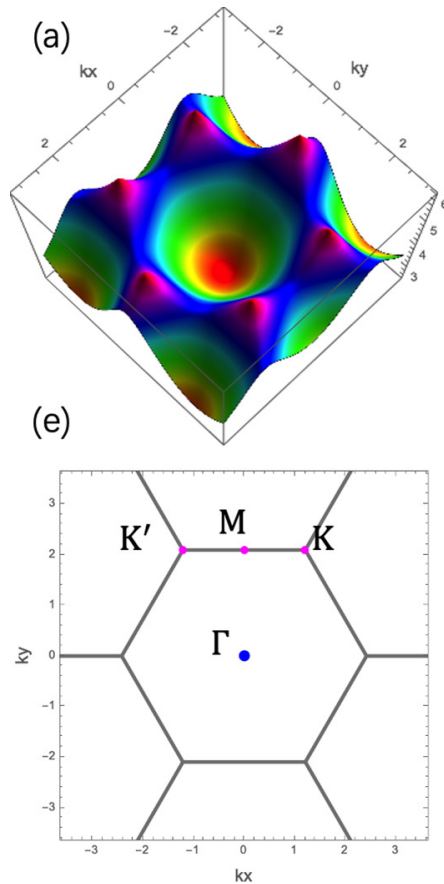

\section{(b)}

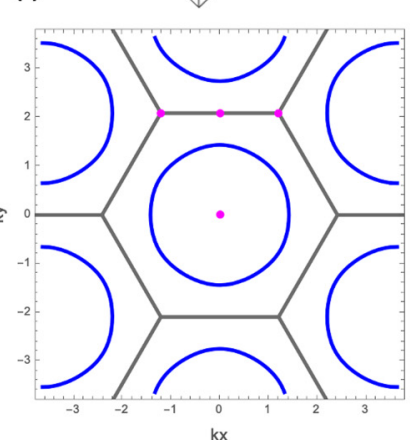

(c)

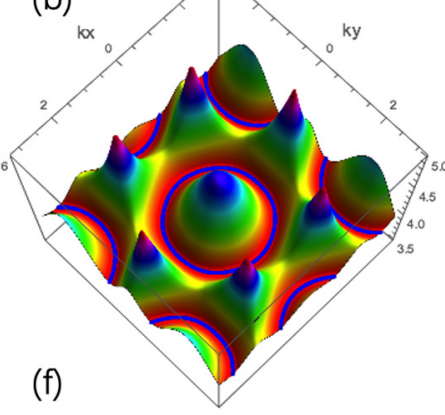

(g)

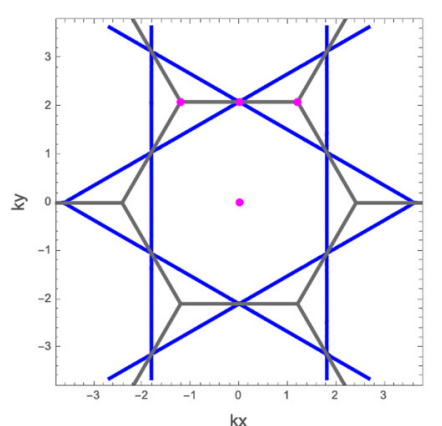

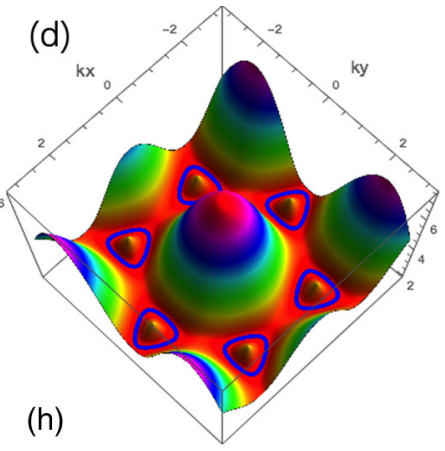

(h)

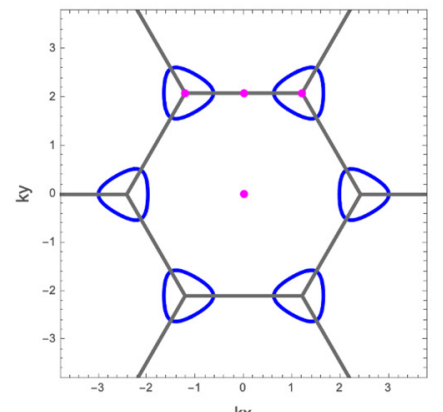

FIG. 2. (Top) The magentic excitation $\omega_{-}(k)$ in the $k_{x}-k_{y}$ plane of the quantum paramagnet with different parameters. (Bottom) The degenerate excitation minima (blue) depicted in the Brillouin Zone (gray). High-symmetry points are marked as pink. We set $D_{z}=6 J_{1}$ in the figure, and $J_{2} / J_{1}$ takes the following parameters [(a) and (e)] $J_{2}=0.1 J_{1}$, [(b) and (f)] $J_{2}=0.3 J_{1}$, [(c) and (g)] $J_{2}=0.5 J_{1}$, and [(d) and (h)] $J_{2}=0.7 J_{1}$.

the frustration that is introduced by the competing $J_{1}-J_{2}$ interaction. With only first-neighbor $J_{1}$ interaction, the exchange part is simply the nearest-neighbor Heisenberg model on the bipartite honeycomb lattice and is thus not frustrated. With only second neighbor $J_{2}$ interaction, the exchange part is the nearest-neighbor Heisenberg model on the triangular lattice of each sublattice and is known to be not very frustrated. In the intermediate $J_{2} / J_{1}$ and when $J_{2}$ and $J_{1}$ are comparable, a large frustration is expected for the exchange part, and thus we experience a contour degenerate band minima in the excitation spectra. This is a consequence of magnetic frustration on the quantum mechanical excitations of a featureless quantum paramagnetic state.

\section{FRUSTRATED QUANTUM CRITICALITY}

Inside the quantum paramagnetic phase, the interesting aspect occurs in the magnetic excitation spectrum. As the single-ion anisotropy decreases, the exchange part of the interaction becomes more important and controls the ground-state properties of the system. The transition out of the quantum paramagnetic state can be traced by examining the excitation spectrum. As the parameter $D_{z}$ becomes smaller, the gap of the excitation diminishes. At the critical value of $D_{z}$, the gap of the excitation becomes zero and the corresponding lowest energy mode starts to be condensed. This picture works very well for $J_{2} / J_{1} \leqslant 1 / 6$ where the flavor bosons are condensed at the $\Gamma$ point and the system develops an antiferromagnetic order that preserves the translational symmetry. The transition is a conventional $2+1 \mathrm{D} X Y$ transition. For the regime with $J_{2} / J_{1}>1 / 6$, the flavor bosons have a difficulty to find an ordering wave vector to be condensed because of the contour degeneracy for the lowest energy modes. Although the highorder interactions between the flavor bosons would eventually break the degeneracy and select the condensed mode, the presence of the contour degeneracy at the lowest energy modes should control the low-energy physics in the vincinity of the phase transition from the quantum paramagnet. At the phase transition, all these degenerate modes at the bottom of the band become critical at the same time. Thus this is a different kind of critical physics from the conventional one where only discrete numbers of bosonic modes become critical. From the excitation spectra, the low-lying modes have no dispersion on the contour but disperse linearly in the momentum direction normal to the contour. This critical property of the bosons near the degenerate contour looks a bit similar to the fermion modes near the Fermi surface in two spatial dimensions, and we have a constant density of states at low energies. Therefore, when thermal fluctuations are included in the critical regime, we would expect a linear- $T$ heat capacity just like what a Fermi surface would do. In the following, we demonstrate this result explicitly.

The flavor wave theory applies well to the zero-temperature excitations deep inside the quantum paramagnet, but has an obstacle to extend to finite temperatures. Moreover, the renormalization or correction from the high-order interactions may become important when $D_{z}$ gets closer to the critical points and the quantum paramagnet phase becomes unstable. To capture the thermal fluctuation in the critical regime, we turn to a different approach. Because our Hamiltonian has a global $\mathrm{U}(1)$ symmetry, we can map the spin variables into equivalent rotor variables. We introduce an integer-valued operator $n_{i}$ and the $2 \pi$-periodic phase variable $\varphi_{i}$ such that $\left[\varphi_{i}, n_{j}\right]=i \delta_{i j}$. We further identify $S_{i}^{z}$ as $n_{i}$, and $S_{i}^{ \pm}$as $\sqrt{2} e^{ \pm i \varphi_{i}}$. Under this 
identification, we have actually allowed $n_{i}$ to take all integer values instead of $0, \pm 1$ for $S_{i}^{z}$. This extension would not cause any significant effects as the weights of higher integer values are strongly suppressed by the single-ion anisotropy in the Hamiltonian. The spin Hamiltonian now becomes

$$
\begin{aligned}
H_{\text {rotor }}= & \sum_{\langle i j\rangle} J_{1}\left[2 \cos \left(\varphi_{i}-\varphi_{j}\right)+n_{i} n_{j}\right] \\
& +\sum_{\langle\langle i j\rangle\rangle} J_{2}\left[2 \cos \left(\varphi_{i}-\varphi_{j}\right)+n_{i} n_{j}\right] \\
& +\sum_{i} D_{z} n_{i}^{2} .
\end{aligned}
$$

To compute the excitation spectra and obtain the dynamical properties, we implement the coherent state path integral formulation and formally integrate out the variable $n_{i}$. The resulting partition function is

$$
\mathcal{Z}=\int \mathcal{D} \Phi \mathcal{D} \lambda \exp \left[-\mathcal{S}-i \sum_{i} \int_{\tau} \lambda_{i}\left(\left|\Phi_{i}\right|^{2}-1\right)\right],
$$

where the action $\mathcal{S}$ is given as

$$
\begin{aligned}
\mathcal{S} \equiv & \int_{\tau} \sum_{k} \partial_{\tau} \Phi_{\mu, k}^{*}\left(4 D_{z} \mathcal{I}_{2 \times 2}+2 \mathcal{J}_{k}\right)_{\mu \nu}^{-1} \partial_{\tau} \Phi_{\nu, k} \\
& +\sum_{i, j} J_{i j} \Phi_{i}^{*} \Phi_{j} .
\end{aligned}
$$

Here, $J_{i j}$ is the exchange matrix in the position space, and $J_{i j} \equiv J_{1}\left(J_{2}\right)$ if $i j$ is the first (second) neighbor. $\mathcal{J}_{k}$ is the exchange matrix in the reciprocal space. As there are two sublattices, $\mathcal{J}_{k}$ is a $2 \times 2$ matrix and $\mu, v$ are sublattice indices. $I_{2 \times 2}$ is a $2 \times 2$ identity matrix. The complex field $\Phi_{i}$ is identified as $e^{i \varphi_{i}}$, and we have the constraint $\left|\Phi_{i}\right|=1$ on each site that is enforced by the Lagrange multiplier $\lambda_{i}$. To solve for the dynamics, we implement an usual saddle point approximation for the path integral. As the translation symmetry is preserved both in the quantum paramagnetic phase and at the finite temperatures, it is legitimate to assume an uniform ansatz for the Lagrange multiplier $\lambda_{i}$ with $i \lambda_{i} \equiv$ $\beta \Delta(T)$ at the saddle point. By integrating out the $\Phi$ field, we obtain the saddle point equation,

$$
\frac{1}{N} \sum_{i= \pm} \sum_{k} \frac{2 D_{z}+\xi_{i}(k)}{\omega_{i}(k, T)} \operatorname{coth}\left[\frac{\beta \omega_{i}(k, T)}{2}\right]=1,
$$

where $N$ is the total number of the lattice sites, $\omega_{i}(k, T)$ is the excitation spectrum and has a temperature dependence, and $\xi_{i}(k)$ is the eigenvalue of the exchange matrix $\mathcal{J}_{k}$. Here we have

$$
\begin{aligned}
\xi_{i}(k) & =J_{2} \sum_{\mu} \cos \left(k \cdot d_{\mu}\right) \pm\left|J_{1} \sum_{\mu} \exp \left(i k \cdot b_{\mu}\right)\right| \\
& =J_{2}\left[\Lambda^{2}(k)-3\right] \pm J_{1} \Lambda(k)
\end{aligned}
$$

with $\left\{d_{\mu}\right\}$ the six vectors connecting second-neighbor sites, and

$$
\omega_{i}(k, T)=\sqrt{\left[4 D_{z}+2 \xi_{i}(k)\right]\left[\Delta(T)+\xi_{i}(k)\right]} .
$$

The parameter $\Delta(T)$ is solved from the saddle point equation in Eq. (18) for each temperature. Just like the zero-temperature excitation of the quantum paramagnet in the previous section, the low-lying excitation $\omega_{-}(k, T)$ develops the same contour degeneracy at the band minima for the same choice of $J_{2} / J_{1}$.

In the paramagnetic phase both for the finite temperature and zero temperature, the $\Phi$ field is not condensed, and we do not need to single out the condensed piece in Eq. (18). At the zero-temperature phase transition, the spectrum becomes gapless with

$$
\Delta(T=0) \equiv 3 J_{2}+\frac{J_{1}^{2}}{4 J_{2}} .
$$

The critical $D_{z}$ at the transition is obtained by solving the saddle point equation, and we establish the phase boundary between the quantum paramagnet and the ordered ones in the zero-temperature phase diagram of Fig. 1. The critical $D_{z}$ at the phase boundary is nonmonotonic as one increases $J_{2} / J_{1}$ from 0 , and becomes minimal at intermediate $J_{2} / J_{1}$. This indicates the maximal frustration at the intermediate $J_{2} / J_{1}$ regime.

To reveal the critical property in the regime with $J_{2}>J_{1} / 6$ where a contour degenerate critical modes exist, we tune the single-ion anisotropy to the criticality and analyze the saddle point equation. At $D_{z}=D_{z c}$ and finite temperatures, $\Delta(T)$ has a temperature dependence and is defined as

$$
\Delta(T) \equiv \Delta(T=0)+\Delta^{\prime}(T) \equiv \Delta_{0}+\Delta^{\prime}(T) .
$$

The gapless low-lying excitation picks up a self-energy from $\Delta^{\prime}(T)$ and has the form near the band bottom

$$
\begin{aligned}
\omega_{-}(k, T) & \approx \sqrt{\left(4 D_{z c}-2 \Delta_{0}\right) \Delta^{\prime}(T)+v_{\perp, k_{c}}^{2} k_{\perp}^{2}} \\
& \equiv \sqrt{2 A \Delta^{\prime}(T)+v_{\perp, k_{c}}^{2} k_{\perp}^{2}},
\end{aligned}
$$

where we have made a Taylor's expansion at the band bottom, $k_{c}$ is the momentum running along the degenerate contour, $k_{\perp}$ is the momentum component normal to the tangent direction of the degenerate contour, and $v_{\perp, k_{c}}$ is the corresponding speed. The weak temperature dependence of $v_{\perp, k_{c}}$ has been neglected in this expansion. The saddle point equation can be decomposed as

$$
c+\int_{k_{c}, k_{\perp}}^{\Gamma} \frac{A \operatorname{coth}\left[\frac{\beta}{2} \sqrt{2 A \Delta^{\prime}(T)+v_{\perp, k_{c}}^{2} k_{\perp}^{2}}\right]}{\sqrt{2 A \Delta^{\prime}(T)+v_{\perp, k_{c}}^{2} k_{\perp}^{2}}}=1,
$$

where the momentum integration is over the area surrounding the degenerate contour, the constant $c$ is an approximately temperature independent contribution from the integration outside this area and from the $\omega_{+}$branch. At low temperatures, the temperature dependent part of the integration becomes independent of the momentum cutoff $\Gamma$ and depends on $T$ through the dimensionless parameter $A \Delta^{\prime}(T) / T^{2}$. In order for the integration to be constant in the temperature such that the saddle point equation is satisfied, we expect $\Delta^{\prime}(T) \rightarrow T^{2}$ and hence $C_{v} \sim T$ in the limit $T \rightarrow 0$ under this analysis.

Here we make a further remark on the critical property. The enhanced density of the low-energy states is induced by the contour degeneracy for $J_{2}>J_{1} / 6$. Since the contour degeneracy is accidental due to the frustrated interaction, there 
is no symmetry protection for the contour degeneracy. The contour degeneracy will eventually be lifted by fluctuations beyond the analysis above, leading to a modified specific heat behavior at the zero-temperature limit, but this may be difficult to be observed in experiments. For $J_{2} \leqslant J_{1} / 6$, a single critical mode implies a conventional $C_{v} \propto T^{2}$ behavior up to a correction from the fluctuations beyond the current analysis. If one deviates from the critical point, several crossovers and/or transition will appear as one increases the temperatures. This behavior will be discussed in Sec. V.

\section{SPIRAL MAGNETISM FROM QUANTUM ORDER BY DISORDER}

When the exchange part of the Hamiltonian becomes important, the system will eventually develop magnetism. In this regime, we will show that the magnetic frustration plays the traditional role just like what it is conventionally thought. As we expect magnetism, it is legitimate to introduce well-defined order parameters to study the ground-state magnetic properties with a traditional Weiss type of meanfield theory. One could further find the magnetic ordering structures by treating the spin operators as classical vector order parameters and optimizing the mean-field energy. The single-ion anisotropy with a positive $D_{z}$ favors the spins to be ordered in the $x y$ plane. We thus model the magnetic order parameter as

$$
\begin{gathered}
\left\langle\boldsymbol{S}_{i}\right\rangle \equiv m\left[\cos \left(q \cdot r_{i}\right) \hat{x}+\sin \left(q \cdot r_{i}\right) \hat{y}\right], \\
\left\langle\boldsymbol{S}_{j}\right\rangle \equiv m\left[\cos \left(q \cdot r_{i}+\theta_{q}\right) \hat{x}+\sin \left(q \cdot r_{i}+\theta_{q}\right) \hat{y}\right],
\end{gathered}
$$

for $i \in \mathrm{A}$ sublattice and $j \in \mathrm{B}$ sublattice, respectively. Here the offset phase $\theta_{q}$ between two sublattices depends explicitly on the spiral wave vector $q$. The order parameter $m$ depends on the ratio between the single-ion anisotropy and the exchange interaction. For the continuous transition at $D_{z c}$, the order parameter $m$ increases gradually from 0 and becomes maximal at $D_{z}=0$. In contrast, the ordering wave vector $q$ is decided by the "kinetic part," i.e., the exchange interactions. Thus the order parameter $m$ and the ordering wave vector $q$ are separately optimized or determined. It is ready to obtain that the exchange interaction, at the mean-field level, requires the ordering wave vector to satisfy the following conditions. For $J_{2}<J_{1} / 6$, a simple antiferromagnetic Néel state is expected with $q=0$ and $\theta_{q}=\pi$. This state is labeled as Néel $x y$ state in the phase diagram of Fig. 1. For $J_{2}>J_{1} / 6$, there exists a degenerate contour in the reciprocal space where the optimal $q$ 's reside. Remarkably, this degenerate contour for the ordering wave vector $q$ is precisely the degenerate contour that is formed by the band minima of the excitation spectrum in the quantum paramagnetic phases, and we have

$$
\begin{aligned}
& \Lambda(q) \equiv\left|\sum_{\mu} \exp \left(-i q \cdot b_{\mu}\right)\right|=\frac{J_{1}}{2 J_{2}}, \\
& \theta_{q}=\pi+\arg \left[\sum_{\mu} \exp \left(-i q \cdot b_{\mu}\right)\right] .
\end{aligned}
$$

Here the contour degeneracy is not protected by any symmetry of the system and thus is an artifact of the mean-field treatment. It is expected that, once quantum fluctuations beyond the mean-field theory are included, the contour degeneracy will be lifted. This effect is known as quantum order by disorder. To establish the breaking of contour degeneracy, we implement a standard linear spin wave analysis for the candidate spiral magnetic orders with a finite ordering wave vector $q$. This approach not only produces the magnetic ordering structures but also generates the spectrum of the magnetic excitations.

For the spin spiral order that is defined in Eqs. (25) and (26), we rewrite the spin operators by introducing the following Holstein-Primakoff bosons,

$$
\begin{gathered}
S_{i} \cdot \hat{n}_{i}=1-b_{i}^{\dagger} b_{i}, \\
S_{i} \cdot \hat{z}=\frac{\sqrt{2}\left(b_{i}+b_{i}^{\dagger}\right)}{2}, \\
S_{i} \cdot\left(\hat{z} \times \hat{n}_{i}\right)=\frac{\sqrt{2}\left(b_{i}-b_{i}^{\dagger}\right)}{2 i},
\end{gathered}
$$

where $\hat{n}_{i}$ is the orientation of the spin order at site $i$. With this substitution of the spin operators, we obtain the leading spin wave correction to the classical ground-state energy,

$$
\begin{aligned}
H_{\mathrm{sw}}= & \frac{1}{2} \sum_{k \in \mathrm{BZ}}\left(b_{k \mathrm{~A}}^{\dagger}, b_{\bar{k} \mathrm{~A}}, b_{k \mathrm{~B}}^{\dagger}, b_{\bar{k} \mathrm{~B}}\right)\left(\begin{array}{cc}
M_{1} & M_{2} \\
M_{2}^{*} & M_{1}
\end{array}\right)\left(\begin{array}{c}
b_{k \mathrm{~A}} \\
b_{\bar{k} \mathrm{~A}}^{\dagger} \\
b_{k \mathrm{~B}} \\
b_{\bar{k} \mathrm{~B}}^{\dagger}
\end{array}\right) \\
& +C+E_{c l},
\end{aligned}
$$

where the matrices and constants are given as

$$
\begin{aligned}
& M_{1}=\frac{J_{2}}{2} \sum_{\mu} e^{i k \cdot d_{\mu}}\left(\begin{array}{cc}
1+\cos \Theta_{d_{\mu}} & 1-\cos \Theta_{d_{\mu}} \\
1-\cos \Theta_{d_{\mu}} & 1+\cos \Theta_{d_{\mu}}
\end{array}\right) \\
& -I_{2 \times 2}\left(J_{1} \sum_{\mu} \cos \Theta_{b_{\mu}}+J_{2} \sum_{\mu} \cos \Theta_{d_{\mu}}\right) \\
& +D_{z}\left(\begin{array}{ll}
1 & 1 \\
1 & 1
\end{array}\right), \\
& M_{2}=\frac{J_{1}}{2} \sum_{\mu} e^{i k \cdot b_{\mu}}\left(\begin{array}{cc}
1+\cos \Theta_{b_{\mu}} & 1-\cos \Theta_{b_{\mu}} \\
1-\cos \Theta_{b_{\mu}} & 1+\cos \Theta_{b_{\mu}}
\end{array}\right) \text {, } \\
& C=\frac{N}{2}\left(J_{1} \sum_{\mu} \cos \Theta_{b_{\mu}}+J_{2} \sum_{\mu} \cos \Theta_{d_{\mu}}\right), \\
& E_{c l}=\frac{N}{2}\left(J_{1} \sum_{\mu} \cos \Theta_{b_{\mu}}+J_{2} \sum_{\mu} \cos \Theta_{d_{\mu}}\right),
\end{aligned}
$$

with $\Theta_{b_{\mu}} \equiv q \cdot b_{\mu}+\theta_{q}, \Theta_{d_{\mu}} \equiv q \cdot d_{\mu}$, and $\sum_{\mu}$ are over the first-neighbor vectors $\left\{b_{\mu}\right\}$ or the second neighbor vectors $\left\{d_{\mu}\right\}$ of the honeycomb lattice. Here, $E_{c l}$ is the classical ground-state energy of the spiral ordered state. Diagonalizing this linear spin-wave Hamiltonian via a generalized Bogoliubov transformation, we obtain two spin-wave modes $\Omega_{ \pm}(k)$. Accordingly, the zero-point energy is given as

$$
E_{\mathrm{zpt}}=\frac{1}{2} \sum_{k \in \mathrm{BZ}} \sum_{ \pm} \Omega_{ \pm}(k)+C+E_{c l} .
$$

As expected, the quantum energy correction lifts the contour degeneracy when $J_{2}>J_{1} / 6$, and exhibits discrete band min- 


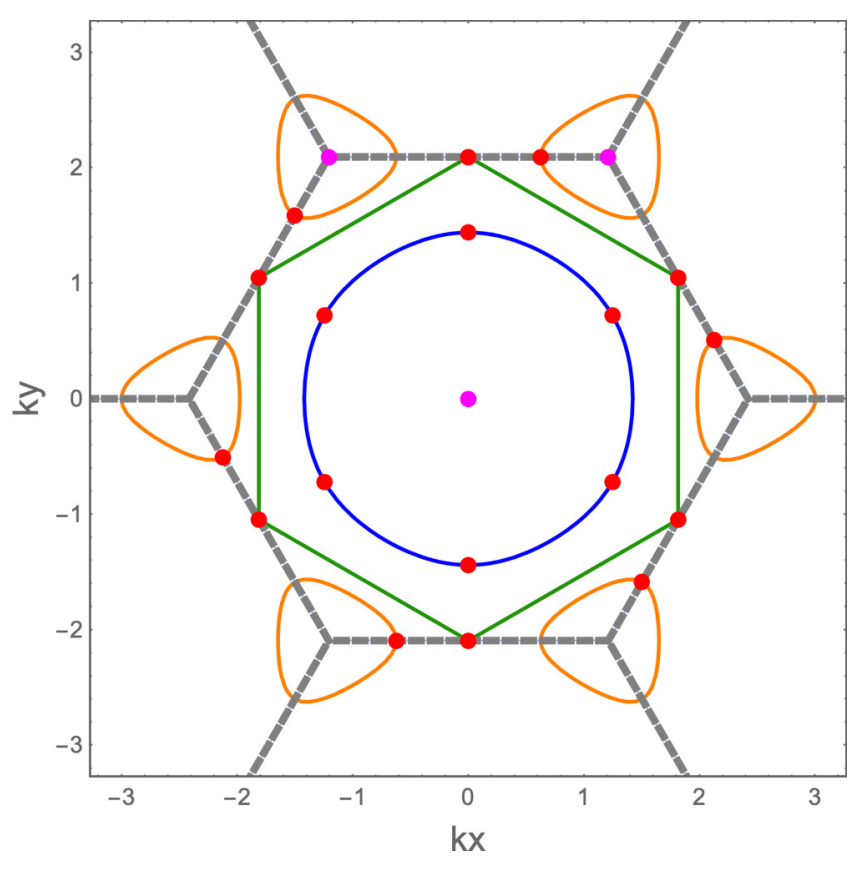

FIG. 3. The evolution of the degenerate contour for the candidate spiral wave vectors for $J_{2} / J_{1}=0.3,0.5,0.7$ from inside to outside. The red points indicate the ordering wave vectors selected by quantum order by disorder. The Brillouin zone boundary is shown in gray.

ima at certain modes. The optimal spiral wave vectors are given by

$\boldsymbol{Q}_{A}=\left(0, \frac{2}{3} \cos ^{-1}\left[\left(\frac{J_{1}}{4 J_{2}}\right)^{2}-\frac{5}{4}\right]\right)$, for $\frac{J_{1}}{6}<J_{2}<\frac{J_{2}}{2}$,

$\boldsymbol{Q}_{B}=\left(\frac{2}{\sqrt{3}} \cos ^{-1}\left(\frac{J_{1}}{4 J_{2}}+\frac{1}{2}\right), \frac{2 \pi}{3}\right), \quad$ for $\quad J_{2}>\frac{J_{1}}{2}$,

and their symmetry equivalents. As long as $D<D_{z c}$, the exact form of classical degenerate contour as well as the quantum selected wave vectors, only depend on the value of $J_{2} / J_{1}$. As we plot in Fig. 3, we illustrate the evolution of degenerate contour and optimal wave vectors as increasing $J_{2} / J_{1}$ from $1 / 6$. When $J_{2} / J_{1}>1 / 6$, the contour emerges, surrounds the $\Gamma$ point, and gradually expands; it then touches the boundary of the Brillouin zone when $J_{2} / J_{1}=1 / 2$; as $J_{2} / J_{1}$ is further increased, the contour surrounds $K$ and $K^{\prime}$ points, and finally it shrinks to $K$ and $K^{\prime}$ points when $J_{2} / J_{1} \rightarrow \infty$, corresponding to the $120^{\circ}$ order on two decoupled triangular lattices. Our results in this section are consistent with the previous studies on the honeycomb lattice $J_{1}-J_{2}$ model without the anisotropy for the generic spins [39] where the contour degeneracy and order-by-disorder were established at $D_{z}=0$. The positive anisotropic $D_{z}$ term in our model simply makes the $x y$ plane as an easy plane to set spins on. The discrete wave vectors selected by the quantum zero point energy are marked by red points in Fig. 3. The resulting two different spiral states characterized by $\boldsymbol{Q}_{A}$ and $\boldsymbol{Q}_{B}$ are denoted as $\operatorname{Spiral}_{x y}^{A}$ and $\mathrm{Spiral}_{x y}^{B}$ in Fig. 1, respectively.

Once the magnetic order is determined from the quantum order by disorder, we proceed to evaluate the magnetic exci- tation with respect to the ordered states. In Fig. 4, we show the magnetic excitations in spin ordered states. For the Néel $x y$ phase, the spin wave excitations are twofold degenerate when $D_{z}=0$, with two Goldstone modes at the $\Gamma$ point, see Fig. 4(a). The presence of $D_{z}$ splits the degeneracy, and only one Goldstone mode is left at the $\Gamma$ point, reflecting the symmetry reducing from $\mathrm{SU}(2)$ to $\mathrm{U}(1)$, see Fig. 4(b). Similar effects occur for the Spiral ${ }_{x y}$ phase, except the relevant Goldstone modes appear at both the $\Gamma$ point and the ordering wave vector, see Figs. 4(c) and 4(d).

\section{DISCUSSION}

Here we discuss various aspects that are related to actual experiments. Probably the most clear indication for the presence of the single-ion anisotropy appears in the magnetic susceptibilities of the single-crystal samples. If one applies the field normal to the honeycomb plane and in the honeycomb plane, our simple mean-field calculation gives two different Curie-Weiss temperatures with

$$
\begin{gathered}
\Theta_{C W}^{z}=-D_{z} / 3-\left(2 J_{1}+4 J_{2}\right), \\
\Theta_{C W}^{\perp}=+D_{z} / 6-\left(2 J_{1}+4 J_{2}\right) .
\end{gathered}
$$

The comparison between these two temperatures could actually approximately give the value of the single-ion anisotropy.

As our model exhibits a global U(1) symmetry, in principle there would exist a Berezinsky-Kosterliz-Thouless transition out of the ordered phases as one increases temperature. However, the actual situation in realistic materials is more complex. The interlayer coupling and other anisotropic interactions would intervene and disrupt or alter this transition. Due to the presence of the contour degeneracy on the ordered sides for $J_{2}>J_{1} / 6$, when the thermal fluctuation smears the difference in the quantum zero point energy for the spiral states from the degenerate contour, a crossover to the quantum critical regime of the frustrated quantum criticality occurs and we expect the results obtained in Sec. III. When thermal fluctuations weaken the quantum effect, the thermal fluctuation would be dominated by the fluctuation modes near the degenerate contour at low temperatures on the ordered side, this paramagnetic regime is sometimes referred as "spiral spin liquid". This is not a new phase of matter but a thermal regime with interesting equal-time spin correlation properties due to the degenerate manifold that governs the low-temperature spin fluctuations because the low-temperature spin fluctuations will be near the degenerate manifold. This can be detected by neutron scattering measurements. Here it is a 2D version, in contrast to the $3 \mathrm{D}$ version with a surface degeneracy on the diamond lattice in Ref. [40]. On the quantum paramagnetic side, the crossover temperature to the critical regime is set by the gap of the magnetic excitations.

A series of materials have been proposed as spin-1 honeycomb lattice magnets [41-47]. These include the layer honeycomb material $\mathrm{BaNi}_{2}\left(\mathrm{XO}_{4}\right)_{2}(X=\mathrm{V}, \mathrm{P}$, and As), the buckled honeycomb material $\mathrm{Ba}_{2} \mathrm{NiTeO}_{6}, \mathrm{Na}_{3} \mathrm{Ni}_{2} \mathrm{BiO}_{6}, \mathrm{Li}_{3} \mathrm{Ni}_{2} \mathrm{SbO}_{6}$, $\mathrm{Na}_{3} \mathrm{Ni}_{2} \mathrm{SbO}_{6}$ and $\mathrm{Ni}_{2} \mathrm{Mo}_{3} \mathrm{O}_{8}$. All of them are Ni-based. The compounds $\mathrm{BaNi}_{2}\left(\mathrm{XO}_{4}\right)_{2}(X=\mathrm{V}, \mathrm{P}$, and As) have been found to have a strong frustration as well as an easy-plane anisotropy. In particular, $\mathrm{BaNi}_{2} \mathrm{~V}_{2} \mathrm{O}_{8}$ is a quasi-2D material 

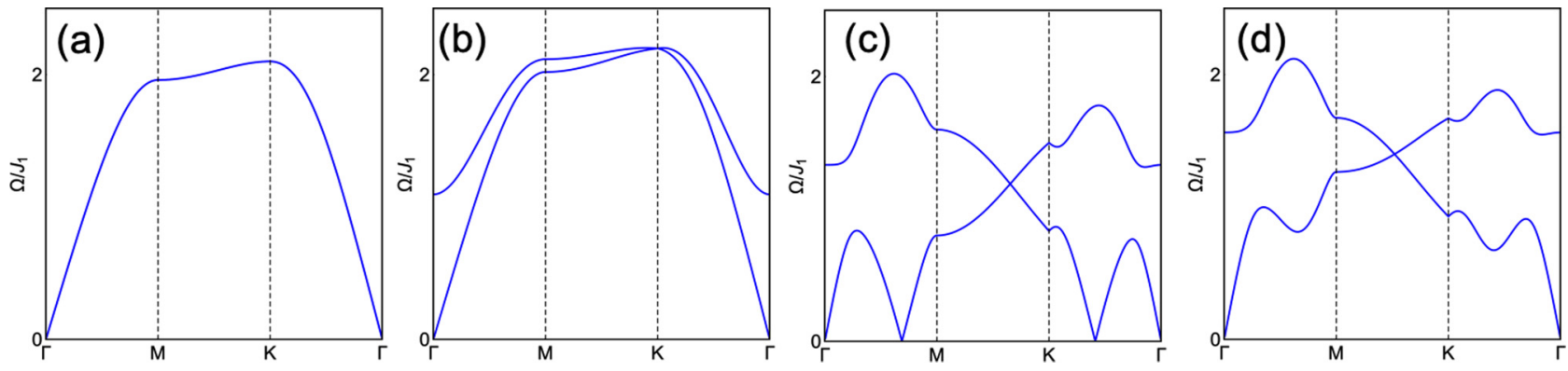

FIG. 4. Representative spin wave excitations along high symmetry momentum lines. (a) $J_{2}=0.1 J_{1}, D_{z}=0 ;\left(\right.$ b) $J_{2}=0.1 J_{1}, D_{z}=0.1 J_{1} ;$ (c) $J_{2}=0.3 J_{1}, D_{z}=0$; and (d) $J_{2}=0.3 J_{1}, D_{z}=0.1 J_{1}$. For (c) and (d), we choose the spin configuration with the wave vector $\boldsymbol{Q}_{A}$ from Eq. (38).

and develop a Néel order. The magnetic excitation has very similar magnetic spectra as shown in Fig. 4(b). While the difference exists in the lowest mode, the spectrum of $\mathrm{BaNi}_{2} \mathrm{~V}_{2} \mathrm{O}_{8}$ detected by inelastic neutron scattering has two gapped modes, rather than one gapped and one gapless mode shown in Fig. 4(b). The gap in the lower mode is explained by the additional easy-axis anisotropy within the honeycomb plane. The magnitude of this easy-axis anisotropy is four order times smaller than the magnitude of exchange interactions such that the spectrum from the inelastic neutron scattering is consistent with our prediction except the small gap in the lowest mode. The easy-axis anisotropy breaks the continuous U(1) symmetry down to $\mathbb{Z}_{2}$ such that the system can develop a long-range magnetic order with a finite temperature phase transition.

In the buckled honeycomb magnet $\mathrm{Ba}_{2} \mathrm{NiTeO}_{6}$ [44], the $\mathrm{Ni}^{2+}$ ions are arranged in the A-B stacking patten and each layer is a triangular lattice. The A-B stacked bilayer is equivalent to a honeycomb lattice. The collinear order that was discovered by neutron diffraction in Ref. [44] is equivalent to the Néel state in Fig. 1. More experiments can be performed on this compound to get more information about the excitation properties.

All three compounds, $\mathrm{Na}_{3} \mathrm{Ni}_{2} \mathrm{BiO}_{6}, \mathrm{Li}_{3} \mathrm{Ni}_{2} \mathrm{SbO}_{6}$ and $\mathrm{Na}_{3} \mathrm{Ni}_{2} \mathrm{SbO}_{6}$, develop antiferromagnetic orders at low temperatures $[46,47] . \mathrm{Na}_{3} \mathrm{Ni}_{2} \mathrm{BiO}_{6}$ actually shows a ferromagnetic Curie-Weiss temperature, indicating competing ferromagnetic and antiferromagnetic interaction. It is possible that the firstneighbor $J_{1}$ interaction here is ferromagnetic and the second neighbor $J_{2}$ interaction is antiferromagnetic. This is captured by the model by flipping the spins from one sublattice. For $\mathrm{Li}_{3} \mathrm{Ni}_{2} \mathrm{SbO}_{6}$ and $\mathrm{Na}_{3} \mathrm{Ni}_{2} \mathrm{SbO}_{6}$, high-field and high-frequency electron spin resonance measurements do provide decisive roles of magnetic anisotropy with polycrystal samples.

The compound $\mathrm{Ni}_{2} \mathrm{Mo}_{3} \mathrm{O}_{8}$ cannot be regarded as a genuine spin-1 honeycomb magnet as the two Ni sublattices experience different crystal field environments [45,48], i.e., an octahedral $\mathrm{Ni}^{2+}$ and a tetrahedral $\mathrm{Ni}^{2+}$. Although the spin part for both sublattices is spin- 1 , the tetrahedral $\mathrm{Ni}^{2+}$ would have an active orbital degree of freedom with a partially filled $t_{2 g}$ level, and as a result, the atomic spin-orbit coupling could then play an important role for the tetrahedral $\mathrm{Ni}^{2+}$ ions and entangle the spin and orbitals. Thus this system is not captured by the model in our work, and a careful modeling requires the involvement of the orbitals on one sublattice.

Finally, we remark on the possibility of multiple- $q$ states that may emerge in the system with magnetic fields and/or at finite temperatures. This kind of states could appear in systems with strong frustration where several equivalent spiral $q$ wave vectors are available [49]. In our model, multiple- $q$ states could appear near the phase boundary between the quantum paramagnet and the spiral ordered side where the system is considering to pick up which $q$ wave vector or several $q$ wave vectors to generate the magnetic order. Likewise, as one cools the system from high temperature paramagnetic phase on the spiral ordered side, the system would experience a similar frustration in choose $q$ wave vectors to generate magnetism. This effect could persist even in the presence of external magnetic fields. The skyrmion lattice may be stabilized with multiple- $q$ states and magnetic fields. These possibilities require an optimization or variational study of the total energy or free energy with respect to the candidate states and are left for future works.

To summarize, we have proposed a generic spin model that incorporates the first and second neighbor exchange interactions and an easy-plane anisotropy in this paper. We established the ground-state phase diagram as plotted in Fig. 1. When the easy-plane anisotropy is much larger compared to exchange interactions, the system lies in a quantum paramagnet phase without any magnetic order, and the magnetic excitation develops a contour degenerate due to the frustration in the exchange interactions. When the exchange interactions are dominant, a Néel or spiral magnetic order is established at zero temperature from the quantum order by disorder. The disordered quantum paramagnet and the ordered Néel or spiral magnetism are separated by a frustrated quantum criticality. Thus the role of frustration has been greatly extended from the ordered side to the disordered one and the associated magnetic excitations and quantum transition.

\section{ACKNOWLEDGMENTS}

This work is supported by research funds from the Ministry of Science and Technology of China with Grants No. 2018YFGH000095, No. 2016YFA0300500, and No. 2016YFA0301001, and from Shanghai Municipal Science and Technology Major Project with Grant No. 2019SHZDZX04, and from the Research Grants Council of Hong Kong with General Research Fund Grants No. 17303819 and No. 17306520. Z.W. is supported by the U.S. Department of Energy, Basic Energy Sciences Grant No. DE-FG0299ER45747. 
[1] G. H. Wannier, Antiferromagnetism. the triangular ising net, Phys. Rev. 79, 357 (1950).

[2] J. Vannimenus and G. Toulouse, Theory of the frustration effect. II. ising spins on a square lattice, J. Phys. C: Solid State Phys. 10, L537 (1977).

[3] L. Balents, Spin liquids in frustrated magnets, Nature (London) 464, 199 (2010).

[4] L. Savary and L. Balents, Quantum spin liquids: A review, Rep. Prog. Phys. 80, 016502 (2016).

[5] P. A. Lee, From high temperature superconductivity to quantum spin liquid: Progress in strong correlation physics, Rep. Prog. Phys. 71, 012501 (2007).

[6] Y. Zhou, K. Kanoda, and T.-K. Ng, Quantum spin liquid states, Rev. Mod. Phys. 89, 025003 (2017).

[7] A. Fert, N. Reyren, and V. Cros, Magnetic skyrmions: Advances in physics and potential applications, Nat. Rev. Mater. 2, 17031 (2017).

[8] Y. Kohama, H. Ishikawa, A. Matsuo, K. Kindo, N. Shannon, and Z. Hiroi, Possible observation of quantum spin-nematic phase in a frustrated magnet, Proc. Natl. Acad. Sci. U.S.A. 116, 10686 (2019).

[9] M. E. Zhitomirsky and H. Tsunetsugu, Magnon pairing in quantum spin nematic, Europhys. Lett. 92, 37001 (2010).

[10] F.-Y. Li, Y.-D. Li, Y. B. Kim, L. Balents, Y. Yu, and G. Chen, Weyl magnons in breathing pyrochlore antiferromagnets, Nat. Commun. 7, 12691 (2016).

[11] H. Kondo, Y. Akagi, and H. Katsura, Three-dimensional topological magnon systems, Phys. Rev. B 100, 144401 (2019).

[12] W. Yao, C. Li, L. Wang, S. Xue, Y. Dan, K. Iida, K. Kamazawa, K. Li, C. Fang, and Y. Li, Topological spin excitations in a threedimensional antiferromagnet, Nat. Phys. 14, 1011 (2018).

[13] S. Bao, J. Wang, W. Wang, Z. Cai, S. Li, Z. Ma, D. Wang, K. Ran, Z.-Y. Dong, D. L. Abernathy, S.-L. Yu, X. Wan, J.-X. Li, and J. Wen, Discovery of coexisting Dirac and triply degenerate magnons in a three-dimensional antiferromagnet, Nat. Commun. 9, 2591 (2018).

[14] C. Castelnovo, R. Moessner, and S. L. Sondhi, Magnetic monopoles in spin ice, Nature (London) 451, 42 (2008).

[15] M. Hermele, M. P. A. Fisher, and L. Balents, Pyrochlore photons: The $U(1)$ spin liquid in a $S=\frac{1}{2}$ three-dimensional frustrated magnet, Phys. Rev. B 69, 064404 (2004).

[16] G. Chen, Dirac's "magnetic monopoles" in pyrochlore ice $U(1)$ spin liquids: Spectrum and classification, Phys. Rev. B 96, 195127 (2017).

[17] T. Senthil, A. Vishwanath, L. Balents, S. Sachdev, and M. P. A. Fisher, Deconfined quantum critical points, Science 303, 1490 (2004).

[18] M. Z. Hasan and C. L. Kane, Colloquium: Topological insulators, Rev. Mod. Phys. 82, 3045 (2010).

[19] X.-L. Qi and S.-C. Zhang, Topological insulators and superconductors, Rev. Mod. Phys. 83, 1057 (2011).

[20] X.-G. Wen, Topological order: From long-range entangled quantum matter to an unification of light and electrons, Condens. Matter Physics 2013, 198710 (2013).

[21] N. Laflorencie, Quantum entanglement in condensed matter systems, Phys. Rep. 646, 1 (2016).

[22] S. Trebst, Kitaev Materials, arXiv:1701.07056.
[23] M. Hermanns, I. Kimchi, and J. Knolle, Physics of the kitaev model: Fractionalization, dynamic correlations, and material connections, Annu. Rev. Condens. Matter Phys. 9, 17 (2018).

[24] H. Takagi, T. Takayama, G. Jackeli, G. Khaliullin, and S. E. Nagler, Concept and realization of Kitaev quantum spin liquids, Nat. Rev. Phys. 1, 264 (2019).

[25] A. Kitaev, Anyons in an exactly solved model and beyond, Ann. Phys. 321, 2 (2006).

[26] P. P. Stavropoulos, D. Pereira, and H.-Y. Kee, Microscopic Mechanism for a Higher-Spin Kitaev Model, Phys. Rev. Lett. 123, 037203 (2019).

[27] F. D. M. Haldane, Nonlinear Field Theory of Large-Spin Heisenberg Antiferromagnets: Semiclassically Quantized Solitons of the One-Dimensional Easy-Axis Néel State, Phys. Rev. Lett. 50, 1153 (1983).

[28] G. Chen, M. Hermele, and L. Radzihovsky, Frustrated Quantum Critical Theory of Putative Spin-Liquid Phenomenology in $6 \mathrm{H}-\mathrm{B}-\mathrm{Ba}_{3} \mathrm{NiSb}_{2} \mathrm{O}_{9}$, Phys. Rev. Lett. 109, 016402 (2012).

[29] G. Chen, Quantum paramagnet and frustrated quantum criticality in a spin-one diamond lattice antiferromagnet, Phys. Rev. B 96, 020412(R) (2017).

[30] F.-Y. Li and G. Chen, Competing phases and topological excitations of spin-1 pyrochlore antiferromagnets, Phys. Rev. B 98 045109 (2018).

[31] C. Xu, F. Wang, Y. Qi, L. Balents, and M. P. A. Fisher, Spin Liquid Phases for Spin-1 Systems on the Triangular Lattice, Phys. Rev. Lett. 108, 087204 (2012).

[32] L. Savary, Quantum loop states in spin-orbital models on the Honeycomb lattice, arXiv:1511.01505.

[33] F. L. Buessen, M. Hering, J. Reuther, and S. Trebst, Quantum Spin Liquids in Frustrated Spin-1 Diamond Antiferromagnets, Phys. Rev. Lett. 120, 057201 (2018).

[34] S. Das, D. Nafday, T. Saha-Dasgupta, and A. Paramekanti, $\mathrm{NiRh}_{2} \mathrm{O}_{4}$ : A spin-orbit entangled diamond-lattice paramagnet, Phys. Rev. B 100, 140408(R) (2019).

[35] J. R. Chamorro, L. Ge, J. Flynn, M. A. Subramanian, M. Mourigal, and T. M. McQueen, Frustrated spin one on a diamond lattice in $\mathrm{NiRh}_{2} \mathrm{O}_{4}$, Phys. Rev. Mater. 2, 034404 (2018).

[36] C. Wang, A. Nahum, and T. Senthil, Topological paramagnetism in frustrated spin-1 mott insulators, Phys. Rev. B 91, 195131 (2015).

[37] Y. Q. Li, M. Ma, D. N. Shi, and F. C. Zhang, Su(4) Theory for Spin Systems with Orbital Degeneracy, Phys. Rev. Lett. 81, 3527 (1998).

[38] A. Joshi, M. Ma, F. Mila, D. N. Shi, and F. C. Zhang, Elementary excitations in magnetically ordered systems with orbital degeneracy, Phys. Rev. B 60, 6584 (1999).

[39] A. Mulder, R. Ganesh, L. Capriotti, and A. Paramekanti, Spiral order by disorder and lattice nematic order in a frustrated heisenberg antiferromagnet on the honeycomb lattice, Phys. Rev. B 81, 214419 (2010).

[40] D. Bergman, J. Alicea, E. Gull, S. Trebst, and L. Balents, Orderby-disorder and spiral spin-liquid in frustrated diamond-lattice antiferromagnets, Nat. Phys. 3, 487 (2007).

[41] E. S. Klyushina, B. Lake, A. T. M. N. Islam, J. T. Park, A. Schneidewind, T. Guidi, E. A. Goremychkin, B. Klemke, and M. Månsson, Investigation of the spin-1 honeycomb antiferromagnet $\mathrm{BaNi}_{2} \mathrm{~V}_{2} \mathrm{O}_{8}$ with easy-plane anisotropy, Phys. Rev. B 96, 214428 (2017). 
[42] L. P. Regnault, J. Y. Henry, J. Rossat-Mignod, and A. De Combarieu, Magnetic properties of the layered nickel compounds $\mathrm{BaNi}_{2}\left(\mathrm{PO}_{4}\right)_{2}$ and $\mathrm{BaNi}_{2}\left(\mathrm{AsO}_{4}\right)_{2}$, J. Magn. Magn. Mater. 15-18, 1021 (1980).

[43] L. P. Regnault, J. P. Boucher, J. Rossat-Mignod, J. Bouillot, R. Pynn, J. Y. Henry, and J. P. Renard, Nonlinear excitations in ld and 2d magnetic systems, Physica B+C 136, 329 (1986).

[44] S. Asai, M. Soda, K. Kasatani, T. Ono, M. Avdeev, and T. Masuda, Magnetic ordering of the buckled honeycomb lattice antiferromagnet $\mathrm{Ba}_{2} \mathrm{NiTeO}_{6}$, Phys. Rev. B 93, 024412 (2016).

[45] J. R. Morey, A. Scheie, J. P. Sheckelton, C. M. Brown, and T. M. McQueen, $\mathrm{Ni}_{2} \mathrm{Mo}_{3} \mathrm{O}_{8}$ : Complex antiferromagnetic order on a honeycomb lattice, Phys. Rev. Mater. 3, 014410 (2019).
[46] J. Werner, W. Hergett, J. Park, C. Koo, E. A. Zvereva, A. N. Vasiliev, and R. Klingeler, The decisive role of magnetic anisotropy in honeycomb layered $\mathrm{Li}_{3} \mathrm{Ni}_{2} \mathrm{SbO}_{6}$ and $\mathrm{Na}_{3} \mathrm{Ni}_{2} \mathrm{SbO}_{6}$, J. Magn. Magn. Mater. 481, 100 (2019).

[47] E. M. Seibel, J. H. Roudebush, H. Wu, Q. Huang, M. N. Ali, H. Ji, and R. J. Cava, Structure and magnetic properties of the $\alpha-\mathrm{NaFeO}_{2}$-type honeycomb compound $\mathrm{Na}_{3} \mathrm{Ni}_{2} \mathrm{BiO}_{6}$, Inorg. Chem. 52, 13605 (2013).

[48] S. Li, V. Loganathan, and A. H. Nevidomskyy, Noncollinear antiferromagnetic order and effect of spin-orbit coupling in spin-1 Honeycomb lattice, arXiv:1906.02215.

[49] T. Okubo, S. Chung, and H. Kawamura, Multiple- $q$ States and the Skyrmion Lattice of the Triangular-Lattice Heisenberg Antiferromagnet under Magnetic Fields, Phys. Rev. Lett. 108, 017206 (2012). 